\title{
PREVALENCE OF HOSPITAL-ACQUIRED INFECTION IN INTENSIVE CARE UNIT: A HOSPITAL-BASED STUDY FROM A TERTIARY CARE HOSPITAL OF BIHAR
}

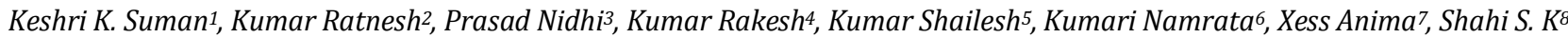 \\ ${ }^{1}$ Senior Resident, Department of Microbiology, Indira Gandhi Institute of Medical Sciences (IGIMS), Patna. \\ ${ }^{2}$ Senior Resident, Department of Microbiology, Indira Gandhi Institute of Medical Sciences (IGIMS), Patna. \\ ${ }^{3}$ Assistant Professor, Department of Microbiology, Indira Gandhi Institute of Medical Sciences (IGIMS), Patna. \\ ${ }^{4}$ Assistant Professor, Department of Microbiology, Indira Gandhi Institute of Medical Sciences (IGIMS), Patna. \\ ${ }^{5}$ Associate Professor, Department of Microbiology, Indira Gandhi Institute of Medical Sciences (IGIMS), Patna. \\ ${ }^{6}$ Associate Professor, Department of Microbiology, Indira Gandhi Institute of Medical Sciences (IGIMS), Patna. \\ 7 Professor, Department of Microbiology, Indira Gandhi Institute of Medical Sciences (IGIMS), Patna. \\ sProfessor, Department of Microbiology, Indira Gandhi Institute of Medical Sciences (IGIMS), Patna.
}

\begin{abstract}
\section{BACKGROUND}

Hospital-Acquired Infection (HAI) have taken on a new dimension with outbreaks of increasingly resistant organisms becoming common. Protocol-based infection control practices in the Intensive Care Unit (ICU) are extremely important. Moreover, baseline information of the prevalence of HAI helps in planning specific implementations of infection control program. The aim of this study was to know the prevalence of Hospital Acquired Infections (HAI) and its isolates with their antibiogram in the medical Intensive Care Unit (ICU) patients in IGIMS, Patna.
\end{abstract}

\section{METHODS}

In our study 187 pts. were suspected having HAI from whom 304 samples from different sources like respiratory samples, urine, blood and pus were collected. The specimens were cultured onto suitable culture media like MacConkey agar, Sheep blood agar, Chocolate agar and Sabouraud Dextrose Agar (SDA). Culture plates were incubated aerobically at $37^{\circ} \mathrm{C}$ for $24-48$ hours.

\section{RESULTS}

Prevalence of HAI in the present study was found to be $18.97 \%$. Out of 304 samples processed, 120 (75.95\%) was bacterial isolates and $38(24.05 \%)$ was fungal isolates. Out of bacterial isolates, Escherichia coli was 28.33\%, and among fugal isolate s yeast other than Candida albicans (YOCA) was 19\%.

\section{CONCLUSIONS}

This study provides baseline information of HAIs and associated risk factors for future surveillance. Efforts are needed to strengthen infection control programs, appropriate national strategies for prevention of HAIs, antibiotic stewardship and repeated prevalence studies in our institution in order to decrease the prevalence of HAI.

\section{KEYWORDS}

Antibiotic Susceptibility, Hospital-Acquired Infections, Medical Intensive Care Unit, Prevalent Microorganisms.

HOW TO CITE THIS ARTICLE: Suman KK, Ratnesh K, Nidhi P, et al. Prevalence of hospital-acquired infection in intensive care unit: a hospital-based study from a tertiary care hospital of Bihar. J. Evolution Med. Dent. Sci. 2016;5(73):5381-5384, DOI: $10.14260 /$ jemds/2016/1221

\section{INTRODUCTION}

Throughout the world, multidrug-resistant hospital-acquired infections are one of the leading causes of death and morbidity among hospitalised patients, accounting a major burden on patients and public health system of any country.[1,2]

The National Nosocomial Infection Surveillance System (NNIS) defines Hospital-Acquired Infection (HAI) as a localised or systemic condition, 1) That results from adverse reaction to the presence of an infectious agent(s) or its toxin(s) and 2) That was not present or incubating at the time

Financial or Other, Competing Interest: None.

Submission 05-08-2016, Peer Review 29-08-2016,

Acceptance 06-09-2016, Published 12-09-2016.

Corresponding Author:

Keshri K. Suman,

Department of Microbiology,

Indira Gandhi Institute of Medical

Sciences (IGIMS),

Patna.

E-mail: keshrisuman45@gmail.com

DOI: $10.14260 /$ jemds $/ 2016 / 1221$ of admission to the hospital. For most bacterial hospitalacquired infections, this means that the infection usually becomes evident 48 hours (i.e. the typical incubation period) or more after admission. However, because the incubation period varies with the type of pathogen and to some extent with the patient's underlying condition, each infection must be assessed individually for evidence that links it to the hospitalisation..$^{[3]}$

The source of HAI may be exogenous (From other patients or the members of the hospital staff or inanimate environment in the hospital) or endogenous (From patient's own flora). In either case, the infecting organism may spontaneously invade the tissues of the patients or may be introduced into them by surgical procedures, instrumental manipulation or nursing procedures. Patients in Intensive Care Units (ICUs) have a higher risk of acquiring HospitalAcquired Infections (HAIs) than those in non-critical care areas. ICU-acquired infection rate is five to ten times higher than hospital-acquired infection rates in general ward patients. ${ }^{1}$ Considerable increased mortality and major costs are associated with ICU-acquired infections. ${ }^{3-5}$ 
Hospital-Acquired Infections (HAI) are a cause of prolonged hospital stay are inconvenient for the patient, constitute an economic burden on health care and increase the mortality of hospitalised patients. ${ }^{4}$ The HAI was defined according to Centres for Disease Control and Prevention Standards. Mechanical ventilation, urinary catheters or intravascular devices were independent risk factors for HAI.6,7

The most common HAI category was urinary tract infection followed by respiratory tract infections and surgical site infection. ${ }^{8}$ Both Gram-positive and Gram-negative bacteria and Candida spp. have been reported as a cause of infection in these patients. Recently, Gram-negative bacilli have been reported more frequently than Gram-positives in this setting. ${ }^{9}$ The ICUs are an area of considerable antibiotic use, in which antibiotic-resistant organisms are prevalent. Their prevalence and rates of resistance can vary enormously depending on geographic location as well as location among ICU types. ${ }^{10}$ For proper management of ICU infections, it is important to have updated knowledge about prevalence of the causative agents and their antimicrobial susceptibility patterns in institution-specific ICUs. ${ }^{11,12}$

The objective of this study was to determine hospitalacquired infection in patients admitted to medical ICU to observe microbial profile in different types of samples and to create guidelines to suggest appropriate empirical antibiotics in HAI based on antibiotic susceptibility pattern of the strain isolated.

\section{MATERIAL AND METHODS}

The present observational study was carried out in the clinical microbiological laboratory of Indira Gandhi Institute of Medial Sciences, Patna, Bihar from December 2011 to June 2013, after the approval of research and Ethics Committee, IGIMS, Patna. A total of 187 patients were studied in the described period from the patients admitted in the 8-bedded Medical Intensive Care Unit (MICU) and 6 bedded Coronary Care Unit (CCU).

\section{Inclusion Criteria}

The patients were observed for any signs of site-specific infections or fever appearing any time after 48 hours of admission into ICU.

\section{Exclusion Criteria}

Patients admitted into ICU with any signs of infections.

Clinical specimens including tracheal aspirates, sputum, urine, urine catheter tips, central venous line tips, blood, body fluids, pus and others were collected from the patients and were processed at Microbiology Laboratory of the hospital. The specimens were cultured onto suitable culture media like MacConkey agar, Sheep blood agar, Chocolate agar and Sabouraud Dextrose Agar (SDA). Culture plates were incubated aerobically at $37^{\circ} \mathrm{C}$ for $24-48$ hours. The isolates were identified by colony morphology, gram-staining and a battery of biochemical reactions. Antimicrobial susceptibility testing was performed on Mueller Hinton agar using disc diffusion method in accordance with Clinical and Laboratory Standard Institute Guidelines (CLSI). Zone size of each antimicrobial agent were recorded and interpreted as resistant, intermediate or susceptible. Intermediately susceptible isolates were considered resistant.

\section{RESULTS}

During our study period, total admissions to our ICU were 1380. Patients admitted for more than 48 hrs. were 580 . Among them 187 pts. were suspected from whom 304 specimens like urine, respiratory samples, blood and pus were collected; 110 pts. were identified to have hospitalacquired infection during their stay in the ICU. Thus, the frequency of Hospital-Acquired Infections (HAI) was $17.64 \%$. Demographic data of patients with Hospital-Acquired infection are summarised in Table 1.

\begin{tabular}{|c|c|c|c|c|}
\hline $\begin{array}{c}\text { Age in } \\
\text { Years }\end{array}$ & No. & \% & $\begin{array}{c}\text { Male } \\
\text { No. (\%) }\end{array}$ & $\begin{array}{c}\text { Female } \\
\text { No. (\%) }\end{array}$ \\
\hline Up to 20 & 2 & $1.8 \%$ & $77(70 \%)$ & $33(30 \%)$ \\
\hline $21-40$ & 16 & $14.5 \%$ & & \\
\hline $41-60$ & 34 & $30.9 \%$ & & \\
\hline $61-80$ & 50 & $45.5 \%$ & & \\
\hline$>80$ & 8 & $7.3 \%$ & & \\
\hline Total & 110 & & & \\
\hline \multicolumn{5}{|c|}{ Pable 1: Demographic Data of } \\
Patients with HAI (N = 110) \\
\hline
\end{tabular}

Out of the 304 specimens processed, 158 samples of 110 patients showed growth after 24-48 hrs. of aerobic incubation are summarised in Table 2.

\begin{tabular}{|c|c|c|}
\hline Samples & Isolates & $\mathbf{\%}$ \\
\hline Urine & 60 & $38 \%$ \\
\hline Respiratory sample & 65 & $41.1 \%$ \\
\hline Blood & 28 & $17.7 \%$ \\
\hline Pus & 5 & $3.2 \%$ \\
\hline \multicolumn{2}{|c|}{$\begin{array}{c}\text { Table 2: No. of Isolates in Different Samples } \\
\text { of Different Patients (N = 110) }\end{array}$}
\end{tabular}

Out of the 110 patients, 30 pts. showed 2 isolates in same or two different samples and 4 pts. were positive for 3 isolates in same or different samples.

\begin{tabular}{|c|c|c|}
\hline Isolates & No. & $\%$ \\
\hline Escherichia coli & 34 & $21.5 \%$ \\
\hline Acinetobacter spp. & 25 & $15.8 \%$ \\
\hline Citrobacter spp. & 19 & $12 \%$ \\
\hline Klebsiella pneumoniae & 12 & $7.6 \%$ \\
\hline Pseudomonas aeruginosa & 10 & $6.3 \%$ \\
\hline $\begin{array}{l}\text { Coagulase negative Staphylococcus spp. } \\
\text { (CONS) }\end{array}$ & 7 & $4.5 \%$ \\
\hline Staphylococcus aureus & 6 & $3.9 \%$ \\
\hline Proteus spp. & 4 & $2.5 \%$ \\
\hline Enterobacter spp. & 1 & $0.6 \%$ \\
\hline Enterococcus spp. & 1 & $0.6 \%$ \\
\hline Streptococcus spp. & 1 & 0.6 \\
\hline Yeast Other than Candida albicans (YOCA) & 30 & $19 \%$ \\
\hline Candida albicans & 8 & $5.1 \%$ \\
\hline \multicolumn{3}{|l|}{$\begin{array}{l}\text { Table 3: Microbial Profile of the } \\
\text { Isolates from Different Samples }\end{array}$} \\
\hline
\end{tabular}

The major pathogens which are responsible for HAI Escherichia coli $(21.5 \%)$, YOCA (19\%), Acinetobacter spp. (15.8\%), Citrobacter spp. (12\%), Klebsiella pneumonia (7.6\%) and Pseudomonas aeruginosa (6.3\%). 
Antimicrobial susceptibility test showed all the isolates of Acinetobacter spp., Klebsiella spp., Citrobacter spp., Pseudomonas aeruginosa, Proteus spp., Staphylococcus aureus and CONS to be resistant to ampicillin. Escherichia coli showed $8.8 \%$ sensitivity to ampicillin. The sensitivity for imipenem in Acinetobacter spp. was 40\%, while in other isolates it was $60-75 \%$. Sensitivity for vancomycin and linezolid among Staphylococcus aureus and CONS was found $80-85 \%$.

\section{DISCUSSION}

In this study we documented an overall HAI prevalence of $18.97 \%$, which was in accordance with other studies particularly from developing countries (13.9\%-17.9\%) (Jroundi et al13 2007; Allegranzi et al14 2011, but less compared to $33.5 \%$ by Beaujean et al,15 but is higher when compared with point prevalence studies from developed countries (5.7\%-6.8\%) (Sartor et al,16 2005). Disease, death and economic costs associated with HAI are increasing, especially in the last few decades. There are certain risk factors associated with high rate of HAIs like length of stay in the hospital, underlying immune compromised conditions, age of the patient, any surgical procedure during hospital stay and presence of invasive medical devices in situ (e.g. vascular catheters, urethral catheters, intubation of the respiratory tract).

Male patients were predominant in the present study and commonest age group affected among both male and female was more than $60-75$ yrs. Malhotra $S$. et $a^{5}$ observed that the infections was more among male than female. The increased incidence in geriatric age group may be due to their defective host defense mechanism was in accordance with other studies like Graves N. et $\mathrm{al}^{2}$ and Malhotra S. et al. ${ }^{5}$ Similarly with respect to the role played by invasive devices in contributing to HAI, present study showed that $38 \%$ of UTI occurred in catheterised patients, $41.10 \%$ of pneumonia was associated with mechanical ventilation, $17.70 \%$ of bloodstream infections with catheter and $3.20 \%$ of local infections could be attributed to the use of invasive devices.

Gram negative organisms commonly reported include Escherichia coli (21.5\%) followed by Acinetobacter spp. (15,8\%), Citrobacter spp. (12\%), Klebsiella pneumonia (7.6\%), Pseudomonas aeruginosa (6.3\%), Proteus spp. (2.5\%), while Gram positive organisms were Coagulase negative Staphylococcus spp. (CONS) (4.5\%), Staphylococcus aureus (3.9\%), Enterococcus spp. and Streptococcus spp. (0.6\%). Among fungal isolate, YOCA (19\%) and Candida albicans (5.1\%) were common isolates. In India, catheters and lung infections are believed to be the primary source of nosocomial infections and gram-negative organisms such as Escherichia coli, Klebsiella, pseudomonas and enterococci are predominant. Candidiasis is also on the rise in intensive care units. ${ }^{16-17}$

Generally, MRSA and multidrug resistant Pseudomonas aeruginosa, Acinetobacter species are known to cause difficulties in the treatment strategies. For the effective eradication of such pathogens can be done by specific higher antibiotics. Many studies have been done on successful elimination of resistant organisms. ${ }^{18}$ Among all other drugs colistin, vancomycin, linezolid and doripenem have given satisfactory results. Colistin therapy in cases of MDR Acinetobacter spp. and P. aeruginosa shown promising results.
In another research, it was found that linezolid is as effective as vancomycin for treating infections caused by MRSA.

Main problems in developing countries are understaffing, poor infrastructure in ICU and poor maintenance of records making situation difficult to get clarity on the incidence of these infections. Although, it is difficult to solve some problems associated with financial hardship in developing countries, most solutions are simple and not resource demanding. Infection control strategies such as hand hygiene and wearing gloves; paying attention to well established processes for decontamination and cleaning of soiled instruments and other items followed by either sterilisation or high-level disinfection and improving safety in operating rooms and other high-risk areas where the most serious and frequent injuries and exposures to infectious agents can resolve the problem to a major extent.

\section{CONCLUSION}

Efforts are needed to strengthen infection control programs, appropriate national strategies for prevention of HAIs, antibiotic stewardship in our institution in order to decrease the prevalence of HAI. Effective infection control measures must be followed in all intensive care units to prevent patients from acquiring infections in the intensive care units.

\section{REFERENCES}

1. Emori TG, Culver DH, Horan TC, et al. National nosocomial infections surveillance system (NNIS): description of surveillance methods. American Journal of Infection Control 1991;19(1):19-35.

2. Kaoutar B, Joly C, L'Heriteau F, et al. Nosocomial infections and hospital mortality: a multicentre epidemiology study. J Hosp Infect 2004;58(4):268-75.

3. Graves N. Economics and preventing hospital-acquired infection. Emerg Infect Dis 2004;10(4):561-6.

4. Shorr AF. Review of studies of the impact on Gramnegative bacterial resistance on outcomes in the intensive care unit. Critical Care Med 2009;37(4):1463-9.

5. Singh $S$, Chaturvedi $R$, Garg SM, et al. Incidence of healthcare associated infection in the surgical ICU of a tertiary care hospital. Medical Journal Armed Forces India 2013;69(2):124 -9.

6. Malhotra S, Sharma S, Hans C. Prevalence of hospital acquired infections in a tertiary care hospital in India. Int Inv J Med Med Sci 2014;1(7):91-94.

7. Mehta A, Rosenthal VD, Mehta Y, et al. Device-associated nosocomial infection rates in intensive care units of seven Indian cities. Findings of the INICC. Journal of Hospital Infection 2007;67(2):168-74.

8. Sharma DK, Tiwari YK, Vyas N, et al. An investigation of the incidence of nosocomial infections among the patients admitted in the intensive care unit of a tertiary care hospital in Rajasthan, India. Int J Curr Microbiol App Sci 2013;2(10):428-35.

9. Kumari HB, Nagarathna S, Chandramuki A. Antimicrobial resistance pattern among aerobic gram-negative bacilli of lower respiratory tract specimens of intensive care unit patients in a neurocentre. Indian J Chest Dis Allied Sci 2007;49(1):19-22.

10. Saleem M, Kumar VA, Zafar IM, et al. Prevalence of nosocomial infections in surgical wards of tertiary care hospital at Lucknow. Ind J Sci Res 2012;3(2):79-84. 
11. The HISI. Newsletter 2007;3(2). http://hisindia.org /data /HISI-Aug07.pdf

12. Thamby SA. A prospective survey and analysis of nosocomial infections in a tertiary care teaching hospital in south India. J Pharm Sci Res 2013;5(11):231-6.

13. Deepti, Sinha S, Sharma SK, et al. Central venous catheter related bloodstream infections in medical intensive care unit patients in a tertiary referral centre. Indian J Chest Dis Allied Sci 2014;56(2):85-91.

14. Jroundi L, Khoudri L, Azzouzi A, et al. Prevalence of hospital-acquired infection in a Moroccan university hospital. Am J Infect Control 2007;35(6):412-6.

15. Allegranzi B, Nejad BS, Combescure C, et al. Burden of endemic health-care-associated infection in developing countries: systematic review and meta-analysis. Lancet 2011;377(9761):228-41.
16. Sartor C, Delchambre A, Pascal L, et al. Assessment of the value of repeated point prevalence surveys for analyzing the trend in nosocomial infections. Infect Control Hosp Epidemiol 2005;26(4):369-73.

17. Safdar N, Crnich CJ, Maki DG. Nosocomial infections in the intensive care unit associated with invasive medical devices. Curr Infect Dis Rep 2001;3(6):487-95.

18. Agarwal R, Gupta D, Ray P, et al. Epidemiology, risk factors and outcome of nosocomial infections in a respiratory intensive care unit in north India. J Infection 2006;53(2):98-105. 